\title{
Establishment of a hybrid risk model to predict major cardiac adverse events in patients with non-ST-elevation acute coronary syndromes
}

\author{
NING ZHANG and WENXIAN LIU \\ Department of Cardiology, Beijing Institute of Heart, Lung and Blood Vessel Disease, Beijing Anzhen Hospital, \\ Capital Medical University, Beijing 100029, P.R. China
}

Received October 27, 2014; Accepted December 1, 2015

DOI: $10.3892 / \mathrm{etm} .2016 .3270$

\begin{abstract}
The present study aimed to generate a hybrid risk model for the prediction of major cardiac adverse events (MACE) in patients with non-ST-elevation acute coronary syndromes (NSTE-ACS), by combining the Global Registry of Acute Coronary Events (GRACE) scoring system and the plasma concentration of $\mathrm{N}$-terminal of the prohormone brain natriuretic peptide (lgNT-proBNP). A total of 640 patients with NSTE-ACS were randomly divided into either the model-establishing group (409 patients) or the prediction model group (231 patients). The clinical endpoint event was MACE, including cardiogenic death, myocardial infarction and heart failure-induced readmission. Among the 409 patients in the model-establishing group, 26 (6.6\%) experienced MACE. The hybrid risk model was calculated using the following equation: Hybrid risk model $=$ GRACE score $+20 \times$ logarithm $(\mathrm{lg})$ NT-proBNP +15 , in which the area under the receiver operating curves (ROCs) for the GRACE score and lgNT-proBNP were 0.807 and 0.798 , respectively. From the equation, the area under the ROC for the hybrid risk model was 0.843 ; thus suggesting that the hybrid risk model may be better able to predict the occurrence of MACE compared with either of its components alone. Following re-stratification, $6 \%$ of patients in the hybrid risk model were re-grouped. A total of 15 patients in the prediction model group experienced MACE (6.5\%). The areas under the ROCs for the hybrid risk model and the GRACE scores for the prediction model group were 0.762 and 0.748 , respectively. The results of the present study suggested that the lgNT-proBNP and GRACE score-established hybrid risk model may improve the accuracy by which MACE are predicted.
\end{abstract}

Correspondence to: Dr Wenxian Liu, Department of Cardiology, Beijing Institute of Heart, Lung and Blood Vessel Disease, Beijing Anzhen Hospital, Capital Medical University, 2 Anzhen Road, Beijing 100029, P.R. China

E-mail: wenxianliucn@126.com

Key words: N-terminal brain peptide precursor, registry and scoring of global acute coronary events, non-ST-elevation acute coronary syndromes, hybrid risk model

\section{Introduction}

Non-ST-elevation acute coronary syndromes (NSTE-ACS) are associated with a higher incidence and mortality rate, as compared with STE-ACS. NSTE-ACS have varying clinical manifestations and prognoses, such that the selection of an appropriate treatment strategy is typically dependent on risk stratification (1). The NSTE-ACS guidelines published by the American College of Cardiology (ACC)/American Heart Association (AHA) and the European Society of Cardiology (ESC) in 2007, recommended that the risk assessment system be used for conducting patient risk stratification (2). At present, the risk scoring system, which was established on the basis of foreign clinical trials and incorporates Thrombolysis in Myocardial Infarction, PURSUIT and Global Registry of Acute Coronary Events (GRACE) scoring systems, is used to conduct risk assessments of NSTE-ACS patients. The current guidelines from the ACC/AHA and ESC are unclear as to which of the three risk scoring systems they recommend; thus suggesting that existing risk stratification methods may discriminate differently between the clinical risks associated with NSTE-ACS $(1,3)$. Among the existing multiple risk scoring systems, GRACE scoring has particular advantages regarding the prediction of cardiovascular events (4), and is currently the most widely used risk scoring system (5-10). However, as compared with the other risk scoring systems, GRACE scoring does not include indices that may reflect neurohumoral factors and hemodynamic alterations; thus its accuracy in predicting cardiovascular events may be limited.

Previous studies have explored the value of combining novel markers and existing risk scoring systems for predicting the clinical risks of NSTE-ACS $(11,12)$. The N-terminal of the prohormone brain natriuretic peptide (NT-proBNP) is a biomarker produced by myocardial cells, and is released into the blood when cardiac cells are stretched or when the wall tension is increased (13). Previous studies have demonstrated the significance of NT-proBNP in heart failure $(14,15)$, and have suggested that NT-proBNP may be used for the risk stratification of ACS $(16,17)$. Guidez et al (11) demonstrated that a hybrid risk scoring system, established from a combination of the plasma BNP concentration and the GRACE scoring system, was able to improve the accuracy of prognostic predictions of patients 
with ACS. This hybrid scoring system was aimed at all patients with ACS; however, to the best of our knowledge, a hybrid risk scoring system has yet to be established for the prediction of cardiac events in patients with NSTE-ACS. The present study aimed to establish a clinical risk prediction model based on a combination of the GRACE scoring system and the logarithm of the plasma NT-proBNP (lgNT-proBNP) concentration, in order to improve the prediction of major cardiac adverse events (MACE), including cardiac death, acute myocardial infarction and heart failure, in patients with NSTE-ACS, and to direct the development of novel treatment strategies.

\section{Materials and methods}

Subjects. A total of 640 patients with NSTE-ACS at the Anzhen Hospital (Beijing, China) were enrolled in the present study between January 2009 and August 2011. Patients were randomly allocated into either the model-establishing group (409 patients, of which 282 were cases of unstable angina and 127 were cases of NSTE-myocardial infarction), or the prediction model group (231 patients, of which 161 were cases of unstable angina, and 70 were cases of NSTE-myocardial infarction). Patients were excluded if they had: i) Acute ST-elevation myocardial infarction; ii) acute and chronic heart failure, with left ventricular ejection fraction $<45 \%$; iii) valvular heart disease; iv) a pacemaker; v) severe liver, kidney or lung diseases; and vi) an infection, cancer or various other conditions. The general characteristics of all patients, including age, gender, height, weight, medical history and situation of current hospitalization, were recorded. The present study was conducted in accordance with the declaration of Helsinki, and with approval from the Ethics Committee of China Capital Medical University (Beijing, China). Written informed consent was obtained from all participants.

Auxiliary examinations. Venous blood was immediately sampled following admission in order to detect markers of myocardial injury, including cardiac troponin 1, creatine kinase and creatine kinase-MB. The fasting blood was sampled in the morning of day 2 following admission, in order to detect various biochemical markers and serum lipids, including NT-proBNP, total cholesterol and low-density lipoprotein cholesterol, and hepatonephric functions via alanine transaminase, aspartate aminotransferase, urea and creatinine levels. Echocardiography was performed throughout hospitalization using a Vivid 7 ultrasound system (GE Healthcare Bio-Sciences, Pittsburgh, PA, USA).

Determination of plasma NT-proBNP concentration. The plasma concentration of NT-proBNP in the venous blood samples collected immediately following admission were measured using a Dimension RxL Max automatic biochecmical analyzer (Siemens AG, Munich, Germany), with a measurable range of $0-30,000 \mathrm{ng} / \mathrm{l}$.

GRACE risk assessment. The GRACE risk assessment was conducted immediately following admission, and included assessment of the following factors: Age, heart rate, systolic blood pressure, serum creatinine levels, cardiac function [as determined by Killip classification (18)], whether the patient was experiencing cardiac arrest upon admission, ST-segment depression and cardiac enzyme elevation (19). GRACE scores of $\leq 88$, between $89-118$, and $>118$ were indicative of a low-, medium- and high-risk of experiencing a MACE, respectively.

Coronary angiography. Coronary angiography was perfected throughout the hospitalization. All patients with unstable angina/acute NSTE-myocardial infarction were treated with the standard secondary drug prevention therapy for coronary heart disease throughout hospitalization, as determined by an experienced physician. These standard therapies included oxygen, $1 \mathrm{ml}$ morphine $(10 \mathrm{mg} / \mathrm{ml}$; Northeast Pharmaceutical Group Co., Ltd., Shenyang, China) for pain relief, anti-platelet therapy and anticoagulation with $2 \mathrm{ml}$ heparin $(12,500 \mathrm{U} / \mathrm{ml}$; Sanofi S.A., Paris, France). A total of $2 \mathrm{ml}$ dopamine $(20 \mathrm{mg} / \mathrm{ml}$; Shanghai Harvest Pharmaceutical Co., Ltd., Shanghai, China) and $2 \mathrm{ml}$ dobutamine $(20 \mathrm{mg} / \mathrm{ml}$; Zhejiang Ruixin Pharmaceutical Co., Ltd., Zhejiang, China) were also administered if systemic vascular resistance was high. Diuretics and fluids were given according to the estimated optimal filling pressures and mechanical ventilation (V60; Maquet Servoi, Berlin, Germany) was applied if necessary.

Follow-up. Follow-up methods included outpatient visits and telephone communication. The average follow-up period was $774 \pm 217$ days.

Statistical analysis. SPSS software, version 16.0 (SPSS, Inc., Chicago, IL, USA) was used to construct the database and perform statistical analyses. Continuous variables were analyzed using the normality test. Data that met the normal distribution initially or following data conversion are presented as the mean \pm standard deviation. Conversely, the non-normally distributed data are presented as the median and interquartile range. Bivariate data were analyzed using linear correlation analysis. $\mathrm{P}<0.05$ was considered to indicate a statistically significant difference. The categorical variables were evaluated using the $\chi^{2}$ test, and the area under the receiver operating characteristic (ROC) curve was used to assess the predictive ability.

Statistical methods used to assess the model-establishing group were as follows: i) lgNT-proBNP was made equal to the magnitude order of GRACE through the standard scores and, according to the correlation coefficient of $1 \mathrm{gNT}$-proBNP and GRACE scoring (0.507), the hybrid risk model was calculated; ii) the hybrid risk model was simplified for clinical application; iii) according to the cutoff point of NT-proBNP, as demonstrated by the ROC curve, NT-proBNP was divided into high- and low-risk groups, which were, along with the original three GRACE scoring groups, regrouped. Based on these regroupings, the hybrid risk model was re-stratified into three groups.

\section{Results}

Determination of NT-proBNP concentration. The NT-proBNP concentration was converted into the logarithmic 
Table I. Baseline data.

\begin{tabular}{|c|c|c|}
\hline Parameter & Model-establishing group & Prediction model group \\
\hline Patients (n) & 409 & 231 \\
\hline Average age (years) & $60 \pm 9$ & $61 \pm 10$ \\
\hline Heart rate (beats/min) & $71 \pm 12$ & $70 \pm 11$ \\
\hline Systolic blood pressure (mmHg) & $132 \pm 18$ & $132 \pm 20$ \\
\hline Males & $284(69.4 \%)$ & $156(67.5 \%)$ \\
\hline History of smoking & $227(55.5 \%)$ & $127(55.0 \%)$ \\
\hline Hypertension & $265(64.8 \%)$ & $165(71.4 \%)$ \\
\hline Diabetes & $132(32.3 \%)$ & $83(35.9 \%)$ \\
\hline Hyperlipidemia & $116(28.4 \%)$ & $162(70.1 \%)$ \\
\hline Serum creatinine $(\mu \mathrm{mol} / \mathrm{l})$ & $83 \pm 26$ & $83 \pm 20$ \\
\hline NT-proBNP (ng/l) & $305.5(1.9,15,070.3)$ & $879.8(6.5,53,900)$ \\
\hline lgNT-proBNP (ng/l) & $1.9 \pm 0.6$ & $2.3 \pm 0.6$ \\
\hline Cardiac troponin I (ng/l) & $3.0(0,92.6)$ & $3.16(0,88.29)$ \\
\hline Creatine kinase (U/1) & $169.5(16,4,874)$ & $210.58(20,5,888)$ \\
\hline Creatine kinase isoenzyme (U/l) & $22.5(0.2,569)$ & $28.53(5,314)$ \\
\hline Total cholesterol (mmol/l) & $4.6 \pm 1.1$ & $4.4 \pm 1.1$ \\
\hline Triglyceride (mmol/l) & $2.8 \pm 0.8$ & $2.7 \pm 0.8$ \\
\hline HDL cholesterol (mmol/l) & $1.1 \pm 0.8$ & $1.1 \pm 0.4$ \\
\hline LDL cholesterol (mmol/l) & $2.8 \pm 0.8$ & $2.7 \pm 0.8$ \\
\hline Hypersensitive C-reactive protein (mg/l) & $4.3(0.08,33.13)$ & $5.7(0.08,43.66)$ \\
\hline GRACE score (points) & $104 \pm 26$ & $112 \pm 34$ \\
\hline Follow-up time (days) & $774 \pm 217$ & $706 \pm 231$ \\
\hline
\end{tabular}

Data are presented as the mean \pm standard deviation or the median (P25, P75). NT-proBNP, N-terminal of the prohormone brain natriuretic peptide; lg, logarithm; HDL, high-density lipoprotein; LDL, low-density lipoprotein; GRACE, global registry of acute coronary events.

value of NT-proBNP (lgNT-proBNP), which exhibited a normal distribution. Similarly, the GRACE scores met the normal distribution. Baseline data are presented in Table I.

Model-establishing group. Among the 409 patients in the model-establishing group, 105, 209 and 95 patients were in the low-, moderate- and high-risk groups, respectively, according to the GRACE scoring system. A total of 26 patients experienced MACE $(6.4 \%)$, including 13 cases of cardiogenic mortality, eight cases of acute myocardial infarction and five cases of heart failure-induced rehospitalization.

Prediction model group. The prediction model group consisted of 231 patients, of which 62,74 and 95 patients were in the low-, moderate- and high-risk groups, respectively, according to the GRACE scoring system. A total of 15 patients experienced MACE $(6.5 \%)$, including three cases of cardiogenic mortality, six cases of acute myocardial infarction and six cases of heart failure-induced rehospitalization.

Association between the NT-proBNP levels and GRACE scores of patients with NSTE-ACS. The correlation between the $\operatorname{lgNT}$-proBNP concentration and GRACE scores was determined using bivariate correlation analysis; the correlation coefficient $(r)$ was 0.507 , which suggests that these two variables are positively correlated $(\mathrm{P}<0.001$; Fig. 1$)$.

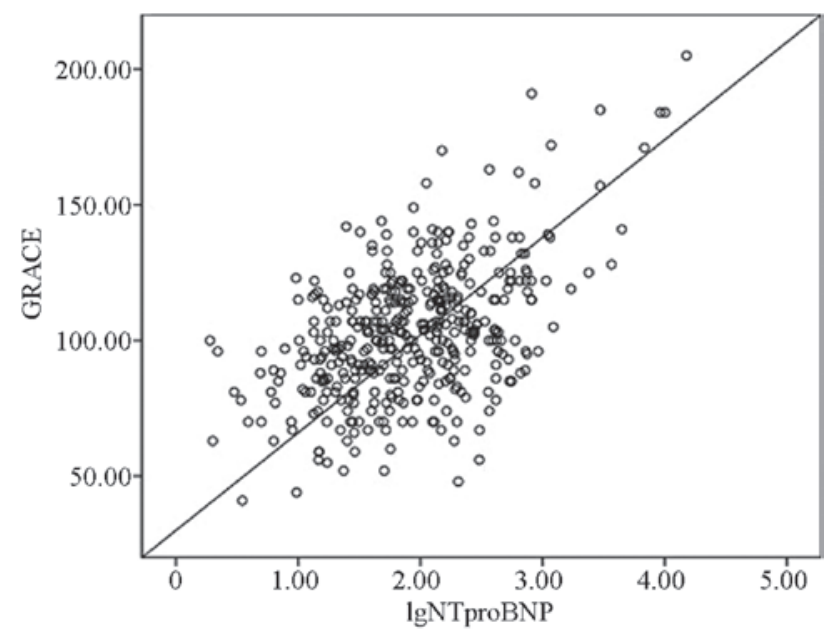

Figure 1. Correlation of the GRACE scores and lgNT-proBNP concentrations from 409 patients with non-ST-elevation acute coronary syndromes in the model-establishing group. GRACE, global registry of acute coronary events; $\operatorname{lgNT}$-proBNP, logarithm of N-terminal of the prohormone brain natriuretic peptide concentration.

Hybrid risk model consists of a combination of the NT-proBNP concentration values and the GRACE scores. The hybrid risk model was established using the following formula: Hybrid risk model $=$ GRACE score $+0.507 \times$ [ 1 gNT-proBNP -1 $.93) / 0.63 \times 24.11+103.54]$. In order to facilitate clinical 
Table II. Comparison of the AUCs of the lgNT-proBNP concentration, GRACE scores and hybrid risk model of 409 NSTE-ACS patients in the prediction model group.

\begin{tabular}{lccc}
\hline Index & AUC & $\mathrm{P}(<0.05)$ & $95 \% \mathrm{CI}$ \\
\hline lgNT-proBNP & 0.798 & 0.000 & $0.740-0.856$ \\
GRACE score & 0.807 & 0.000 & $0.749-0.865$ \\
Hybrid risk model & 0.843 & 0.000 & $0.791-0.895$
\end{tabular}

NSTE-ACS, non-ST elevation acute coronary syndrome; lgNT-proBNP, logarithm of the N-terminal of the prohormone brain natriuretic peptide concentration; GRACE, global registry of acute coronary events; MACE, major adverse cardiac events; AUC, area under the receiver operating characteristic curve.

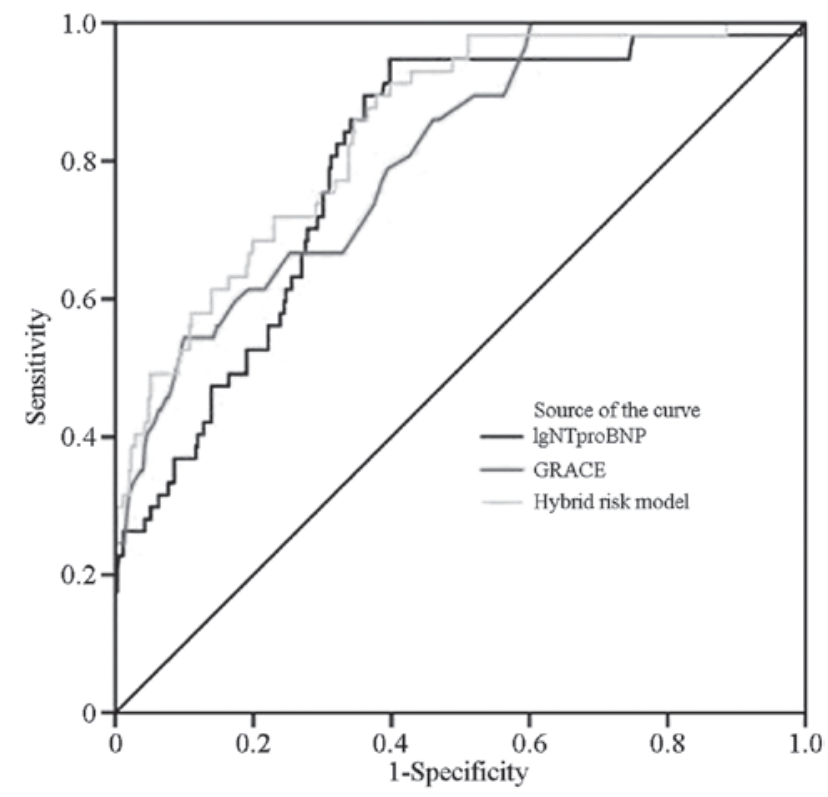

Figure 2. Receiver operating characteristic (ROC) curves for the lgNT-proBNP concentrations, the GRACE scores and the hybrid risk model from 409 patients with non-ST-elevation acute coronary syndromes in the model-establishing group. The areas under the ROC curves are indicative of the ability of the variables/models to predict major adverse cardiac events. lgNT-proBNP, logarithm of the N-terminal of the prohormone brain natriuretic peptide concentration; GRACE, global registry of acute coronary events.

applications, the hybrid risk model was simplified as follows: Hybrid risk model $=$ GRACE score $+20 \times$ lgNT-proBNP +15 . The following analyses were conducted using the simplified risk model. The hybrid risk model was regrouped for the evaluation of NT-pro BNP, which generated new risk stratifications as follows: Low risk, $<135$ points; moderate-risk, $135-170$ points; and high-risk, $>170$ points. Each risk layer of the hybrid risk model underwent the $\chi^{2}$ test $\left(\chi^{2}=58.494 ; \mathrm{V}=2\right.$; $\mathrm{P}=0.000$ ), which suggested that there were significant differences in the incidence of MACE among these three groups. Following re-stratification, seven cases in the high-risk group were downgraded to the moderate-risk group, eight cases from the high-risk group were downgraded to the low-risk group, and ten cases in the low-risk group were upgraded to the high-risk group; therefore $6 \%$ of all patients were re-grouped. In addition, among the 15 patients that experienced MACE, two were upgraded from the moderate-risk group to the high-risk group; thus accounting for $8 \%$ of patients.

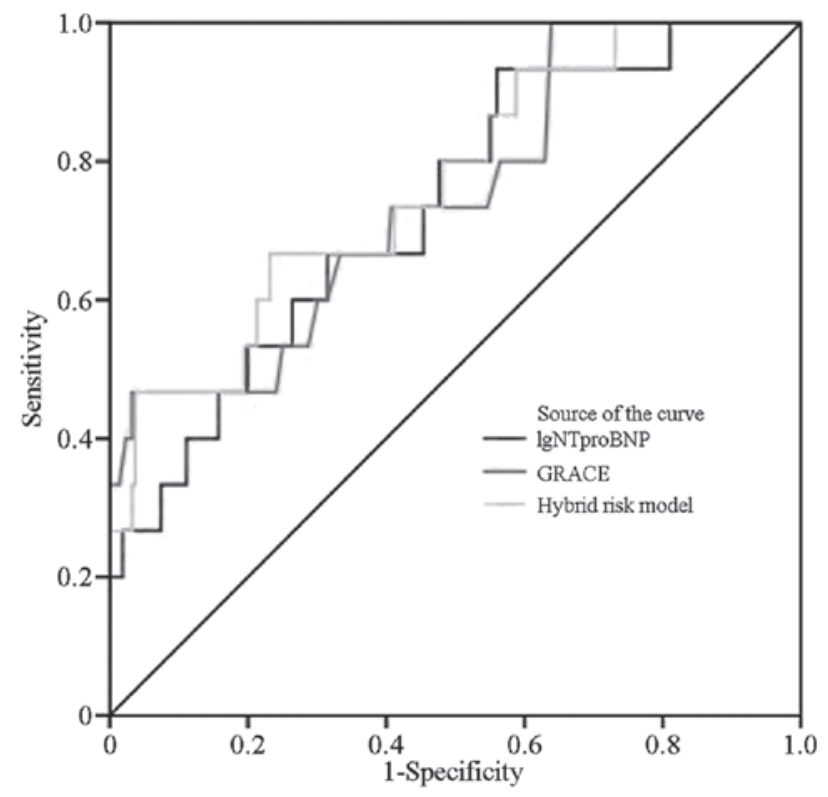

Figure 3. Receiver operating characteristic (ROC) curves for the lgNT-proBNP concentrations, the GRACE scores and the hybrid risk model from 231 patients with non-ST-elevation acute coronary syndromes in the prediction model group. The areas under the ROC curves are indicative of the ability of the variables/models to predict major adverse cardiac events. lgNT-proBNP, logarithm of N-terminal of the prohormone brain natriuretic peptide concentration; GRACE, global registry of acute coronary events.

Areas under the ROC curves of the NT-proBNP concentrations, GRACE scores and the hybrid risk model indicate an ability to predict MACE.

Model-establishing group. The area under the ROC curves for the lgNT-proBNP concentrations and GRACE scores of the model-establishing group were $0.798(\mathrm{P}=0.000)$ and $0.807(\mathrm{P}=0.000)$, respectively. These results suggested that both methods are able to predict MACE. The area under the ROC curve for the hybrid risk model was significantly increased (0.843), as compared with those of the GRACE score and the lgNT-proBNP concentration $(\mathrm{P}<0.05$; Fig. 2; Table II); thus suggesting that the hybrid risk model has a greater predictive ability, as compared with either of its constituents.

Prediction model group. The area under the ROC curve of the hybrid risk model of the prediction model group was 0.762 , which was significantly increased, as compared with that of the GRACE score $(0.748 ; \mathrm{P}<0.05$; Fig. 3; Table III). 
Table III. Comparison of AUCs of the lgNT-proBNP concentration, GRACE scores and hybrid risk model of 231 NSTE-ACS patients in the prediction model group.

\begin{tabular}{lccc}
\hline Index & AUC & $\mathrm{P}(<0.05)$ & $95 \% \mathrm{CI}$ \\
\hline lgNT-proBNP & 0.734 & 0.002 & $0.606,0.862$ \\
GRACE score & 0.748 & 0.001 & $0.615,0.881$ \\
Hybrid risk model & 0.762 & 0.001 & $0.633,0.891$ \\
\hline
\end{tabular}

NSTE-ACS, non-ST elevation acute coronary syndrome; lgNT-proBNP, logarithm of the N-terminal of the prohormone brain natriuretic peptide concentration; GRACE, global registry of acute coronary events; MACE, major adverse cardiac events; AUC, area under the receiver operating characteristic curve.

\section{Discussion}

Risk stratification has an important role in the evaluation and management of patients with ACS, since these patients exhibit varying prognoses. The existing guidelines recommend that patients should undergo a risk assessment upon hospital admission $(20,21)$; thus suggesting that timely and accurate risk stratification may go towards improving the prognosis of a patient. The brain natriuretic peptide (BNP) is a biomarker that is rapidly released into the blood when myocardial cells are stretched or the wall tension is increased (13). BNP and NT-proBNP are derived from the same source and are secreted in equimolar concentrations. NT-proBNP is more stable in vitro and has a longer half-life, as compared with BNP; thus, the present study selected NT-proBNP for incorporation into the hybrid risk model. Previous studies have demonstrated an association between the levels of BNP and heart failure; myocardial ischemia led to a decrease in myocardial contractility and increased wall tension, which in turn initiated the release of BNP from myocardial cells.

In the model-establishing group in the present study, ROC curves for the $\lg$ NT-proBNP concentrations and GRACE scores were prepared, in order to compare the abilities of these variables to predict MACE. The areas under the ROC curves were $>0.7$; thus suggesting that both variables were able to independently predict MACE. In addition, the $\operatorname{lgNT}$-proBNP concentration was positively correlated with the GRACE score in the model-establishing group $(r=0.507$; $\mathrm{P}<0.001)$. The hybrid risk model was obtained by combining $\operatorname{lgNT}$-proBNP with the GRACE score, and was simplified to the following equation: Hybrid risk model $=$ GRACE score $+20 \times \operatorname{lgNT}$-proBNP +15 . The ROC curve of the simplified hybrid risk model overlapped with that of the un-simplified model (both $\mathrm{AUC}=0.762$ ); thus suggesting that the simplified and un-simplified hybrid risk model had identical clinical values, and that the simplified hybrid risk model may replace the un-simplified hybrid risk model for clinical applications.

In the prediction model group, the area under the ROC curve of the hybrid risk model was 0.762 , which was significantly increased, as compared with that of the GRACE score (0.748; $\mathrm{P}=0.001)$. These results suggested that the hybrid risk model may be better able to predict MACE, as compared with the GRACE score alone. Following re-stratification, seven cases in the high-risk group of the model-establishing group were downgraded to the moderate-risk group, eight cases of the high-risk group were downgraded to the low-risk group, and 10 cases of the low-risk group were upgraded to the high-risk group; thus $6 \%$ of patients were re-grouped. Among the 26 MACE cases, one case in the moderate-risk group was upgraded to the high-risk group, and three cases in the low-risk group were upgraded to the moderate-risk group; thus $15 \%$ of MACE cases were re-grouped. The results of the present study suggested that the hybrid risk model was able to reduce the number of patients in the high-risk group that did not experience MACE, reduce the number of patients in the low-risk group that experienced MACE, and improve the predictive ability of MACE. These results were consistent with a previous study involving 248 patients with ACS, which similarly combined NT-proBNP and the GRACE scores (17); however, since this study only involved ACS patients, and the sample size for model establishment was small, there existed certain limitations. In particular, the incidences and mortality rates of NSTE-ACS have been shown to be higher, as compared with STE-ACS; therefore, NSTE-ACS should be considered separately when generating risk models for the prediction of MACE.

The present study aimed to establish a hybrid risk model for the prediction of MACE in patients with NSTE-ACS, by combining the GRACE scoring system and the lgNT-proBNP concentration. The NT-proBNP concentration has previously been associated with patient prognosis; the plasma concentration of NT-proBNP was demonstrated to be predictive of the prognosis of a patient with heart failure, stable angina and ACS (22). In addition, NT-proBNP has been shown to be a marker of heart failure; therefore, cardiac insufficiency was excluded from the inclusion criteria, in order to reduce its impact on NT-proBNP concentrations. Numerous previous studies have reported that patients with ACS and cardiac insufficiency (Killip grading, grade 2) had a high mortality rate (13,23-25). As compared with the GRACE scores of such indicators as Killip grading and cardiac enzymes, NT-proBNP was demonstrated to be an independent risk factor of ACS in the present study. Furthermore, NT-proBNP was able to predict the prognosis of patients with ACS in a way that was more convenient and cheaper, as compared with the echocardiogram; therefore it may be beneficial to measure plasma NT-proBNP concentration on patient admission. However, the addition of the NT-proBNP concentration into a new risk model has been challenging, and required further study by professional cardiovascular researchers $(26,27)$.

In conclusion, the plasma NT-proBNP concentration and the GRACE scoring system are independent risk factors of MACE that, when combined into a hybrid risk model in the present study, showed improved accuracy for the prediction of MACE in patients with NSTE-ACS.

\section{Acknowledgements}

The authors would like to thank Ms Weihong He and Mr Li Sheng (both Capital Medical University) for their contribution to the present study. 


\section{References}

1. Roffi M, Patrono C, Collet JP, Mueller C, Valgimigli M, Andreotti F, Bax JJ, Borger MA, Brotons C, Chew DP and Gencer B: 2015 ESC Guidelines for the management of acute coronary syndromes in patients presenting without persistent ST-segment elevation: Task force for the management of acute coronary syndromes in patients presenting without persistent ST-segment elevation of the European Society of Cardiology (ESC). Eur Heart J 2015: ehv320.

2. Anderson JL, Adams CD, Antman EM, Bridges CR, Califf RM, Casey DE Jr, Chavey WE II, Fesmire FM, Hochman JS, Levin TN, et al; American College of Cardiology; American Heart Association Task Force on Practice Guidelines (Writing Committee to Revise the 2002 Guidelines for the Management of Patients With Unstable Angina/Non-ST-Elevation Myocardial Infarction); American College of Emergency Physicians; Society for Cardiovascular Angiography and Interventions; Society of Thoracic Surgeons; American Association of Cardiovascular and Pulmonary Rehabilitation; Society for Academic Emergency Medicine: ACC/AHA 2007 guidelines for the management of patients with unstable angina/non-ST-elevation myocardial infarction: A report of the American College of Cardiology/American Heart Association task force on practice guidelines (writing committee to revise the 2002 guidelines for the management of patients with unstable angina/non-ST-elevation myocardial infarction) developed in collaboration with the American College of Emergency Physicians, the Society for Cardiovascular Angiography and Interventions, and the Society of Thoracic Surgeons endorsed by the American Association of Cardiovascular and Pulmonary Rehabilitation and the Society for Academic Emergency Medicine. J Am Coll Cardiol 50: e1-e157, 2007.

3. Amsterdam EA, Wenger NK, Brindis RG, Casey DE Jr, Ganiats TG, Holmes DR Jr, Jaffe AS, Jneid H, Kelly RF, Kontos MC, et al; American College of Cardiology; American Heart Association Task Force on Practice Guidelines; Society for Cardiovascular Angiography and Interventions; Society of Thoracic Surgeons; American Association for Clinical Chemistry: 2014 AHA/ACC guideline for the management of patients with non-ST-elevation acute coronary syndromes: A report of the American College of Cardiology/American Heart Association Task Force on Practice Guidelines. J Am Coll Cardiol 64: e139-228, 2014.

4. Khalill R, Han L, Jing C and Quan H: The use of risk scores for stratification of non-ST elevation acute coronary syndrome patients. Exp Clin Cardiol 14: e25-e30, 2009.

5. Granger CB, Goldberg RJ, Dabbous O, Pieper KS, Eagle KA Cannon CP, Van De Werf F, Avezum A, Goodman SG, Flather MD, et al: Predictors of hospital mortality in the global registry of acute coronary events. Arch Intern Med 163: 2345-2353, 2003.

6. Eagle KA, Lim MJ, Dabbous OH, Pieper KS, Goldberg RJ, Van de Werf F, Goodman SG, Granger CB, Steg PG, Gore JM, et al: A validated prediction model for all forms of acute coronary syndrome: Estimating the risk of 6-month postdischarge death in an international registry. JAMA 291: 2727-2733, 2004.

7. Kozieradzka A, Kamiński KA, Maciorkowska D, Olszewska M, Dobrzycki S, Nowak K, Kralisz P, Prokopczuk P and Musial WJ: GRACE, TIMI, Zwolle and CADILLAC risk scores - do they predict 5-year outcomes after ST-elevation myocardial infarction treated invasively. Int J Cardiol 148: 70-75, 2011.

8. Lindahl B, Toss H, Siegbahn A, Venge P and Wallentin L: Markers of myocardial damage and inflammation in relation to long-term mortality in unstable coronary artery disease. FRISC study group. Fragmin during instability in Coronary artery disease. N Engl J Med 343: 1139-1147, 2000

9. Morrow DA, Antman EM, Giugliano RP, Cairns R, Charlesworth A, Murphy SA, de Lemos JA, McCabe CH and Braunwald E: A simple risk index for rapid initial triage of patients with ST-elevation myocardial infarction: An In TIME II substudy. Lancet 358: 1571-1575, 2001.

10. Meune C, Drexler B, Haaf P, Reichlin T, Reiter M, Meissner J, Twerenbold R, Stelzig C, Freese M, Winkler K and Mueller C: The GRACE score's performance in predicting in-hospital and 1-year outcome in the era of high-sensitivity cardiac troponin assays and B-type natriuretic peptide. Heart 97: 1479-1483, 2011.
11. Guidez T, Maréchaux S, Pincon C, Lamour H, Barrailler S, Decourcelle V, Braun S, Bouabdallaoui N, Bauchart JJ, Auffray JL, et al: Addition of B-type natriuretic peptide to the GRACE score to predict outcome in acute coronary syndrome: A retrospective (development) and prospective (validation) cohort-based study. Emerg Med J 29: 274-279, 2012.

12. Ang DS, Wei L, Kao MP, Lang CC and Struthers AD: A comparison between B-type natriuretic peptide, global registry of acute coronary events (GRACE) score and their combination in ACS risk stratification. Heart 95: 1836-1842, 2009.

13. Bosseau C, Galli E and Donal E. Prognostic value of BNP in heart failure with preserved or reduced ejection fraction. Heart 101: $1855-1856,2015$.

14. Gong H, Wang X, Ling Y, Shi Y and Shi H: Prognostic value of brain natriuretic peptide in patients with heart failure and reserved left ventricular systolic function. Exp Ther Med 7: 1506-1512, 2014.

15. Tate S, Griem A, Durbin-Johnson B, Watt C and Schaefer S: Marked elevation of B-type natriuretic peptide in patients with heart failure and preserved ejection fraction. J Biomed Res 28: 255-261, 2014.

16. Romel SM, Faruque M, Bari MA, Bari MS, Aditya GP, Choudhury AK, Khalequzzaman M, Alam MM, Razzaque MA, Rahman MA, et al: Association between elevated B-Type natriuretic peptide levels with extent of coronary artery disease in patients with unstable angina and NSTEMI. Mymensingh Med J 23: 544-551, 2014.

17. Goyal BM, Sharma SM and Walia M: B-type natriuretic peptide levels predict extent and severity of coronary artery disease in non-ST elevation acute coronary syndrome and normal left ventricular function. Indian Heart J 66: 183-187, 2014.

18. Granger CB, Goldberg RJ, Dabbous O, Pieper KS, Eagle KA, Cannon CP, Van De Werf F, Avezum A, Goodman SG, Flather MD and Fox KA; Global Registry of Acute Coronary Events Investigators: Predictors of hospital mortality in the global registry of acute coronary events. Arch Intern Med 163: 2345-2353, 2003.

19. Fox KA, Dabbous OH, Goldberg RJ, Pieper KS, Eagle KA, Van de Werf F, Avezum A, Goodman SG, Flather MD, Anderson FA Jr and Granger CB: Prediction of risk of death and myocardial infarction in the six months after presentation with acute coronary syndrome: Prospective multinational observational study (GRACE). BMJ 333: 1091,2006

20. Pollack CV Jr and Braunwald E: 2007 update to the ACC/AHA guidelines for the management of patients with unstable angina and non-ST-segment elevation myocardial infarction: Implications for emergency department practice. Ann Emerg Med 51: 591-606, 2008.

21. Bassand JP, Hamm CW, Ardissino D, Boersma E, Budaj A, Fernandez-Aviles F, Fox KA, Hasdai D, Ohman EM, Wallentin L, et al: Guidelines for the diagnosis and treatment of non-ST-segment elevation acute coronary syndromes. Rev Port Cardiol 27: 1063-1143, 2008 (In Portuguese).

22. Omland T, Sabatine MS, Jablonski KA, Rice MM, Hsia J, Wergeland R, Landaas S, Rouleau JL, Domanski MJ, Hall C, et al: Prognostic value of B-Type natriuretic peptides in patients with stable coronary artery disease: The PEACE Trial. J Am Coll Cardiol 50: 205-214,2007.

23. Killip T III and Kimball JT: Treatment of myocardial infarction in a coronary care unit. A two year experience with 250 patients. Am J Cardiol 20: 457-464, 1967.

24. Fathy SA, Abdel Hamid FF, Zabut BM, Jamee AF, Ali MA and Abu Mustafa AM: Diagnostic utility of BNP, corin and furin as biomarkers for cardiovascular complications in type 2 diabetes mellitus patients. Biomarkers 20: 460-469, 2015.

25. Salama R, El-Moniem A, El-Hefney N and Samor T: N-terminal pro-BNP in acute coronary syndrome patients with ST elevation (STE-ACS) versus non ST elevation (NSTE-ACS). Int J Health Sci (Qassim) 5(2 Suppl 1): 27-29, 2011.

26. Møller JE, Pellikka PA, Hillis GS and Oh JK: Prognostic importance of diastolic function and filling pressure in patients with acute myocardial infarction. Circulation 114: 438-444, 2006.

27. Richardson-Lobbedez M, Maréchaux S, Bauters C, Darchis J, Auffray JL, Bauchart JJ, Aubert JM, LeJemtel TH, Lesenne M, Van Belle E, et al: Prognostic importance of tissue Doppler-derived diastolic function in patients presenting with acute coronary syndrome: A bedside echocardiographic study. Eur J Echocardiogr 9: 594-598, 2008. 